\title{
Component Development for Nuclear Hybrid Energy Systems
}

\author{
M. Scott Greenwood ${ }^{1}$ \\ ${ }^{1}$ Oak Ridge National Laboratory, USA, greenwoodms@ornl.gov
}

\begin{abstract}
A Nuclear Hybrid Energy System (NHES) uses a nuclear reactor as the basic power generation unit. The power generated is then used by multiple customers as either thermal power, electrical power, or both. The definition and architecture of an NHES can be adapted based on the needs and opportunities of a given local market. For example, locations in need of potable water may be best served by coupling a desalination plant to the NHES. Similarly, a location near an oil refinery may have a need for emission-free hydrogen production. Using the flexible, multi-domain capabilities of Modelica, Argonne National Laboratory, Idaho National Laboratory (INL), and Oak Ridge National Laboratory (ORNL) are investigating the dynamics (e.g., thermal hydraulics and electrical generation/consumption) and cost of such a hybrid system. This paper examines ongoing NHES work including the modeling organizational layout, highlighting a few subsystems, describing some of the component development and providing results from a study of multi-dimensional conduction model development.
\end{abstract}

Keywords: thermal hydraulic, nuclear, economics, hybrid systems

\section{Introduction}

Electricity markets in the United States are undergoing significant shifts in the traditional market structure. Factors such as mandates for renewable energy, overall carbon reduction, and the emergence of cheap natural gas have strained the profitability of primary baseload electricity suppliers, including nuclear power plants.

As the typical nuclear power generating station traditionally has only one customer - the griddiversification of the customer portfolio in an integrated or hybrid manner may be advantageous. A representative NHES is depicted in Figure 1.

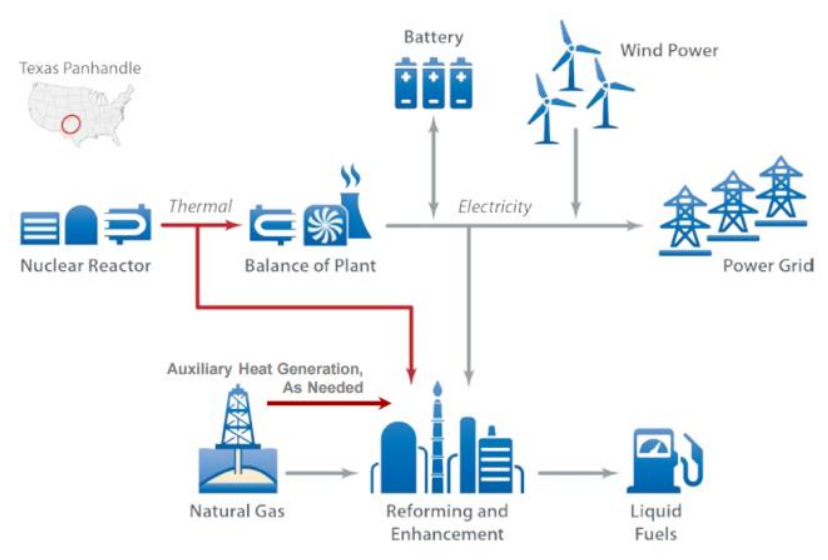

Figure 1. A representative NHES demonstrating a possible coupling scenario of both thermal and electrical energy with additional systems (e.g., an industrial process and energy storage system) (Bragg-Sitton et al. 2015).

A hybrid energy system approach, coupling base load energy suppliers and energy customers (thermal and/or electric), may be profitable and preferred in future energy markets. Possible scenarios include producing products that are more profitable than electricity or mitigating the possible load-following need-and subsequent cost increases - that significant renewable penetration may impose on nuclear power plants. For example, Figure 2 is a representative summary of the Electric Power Research Institute's (EPRI) recent study on the impact of renewable energy generation on grid variability (EPRI, 2015). Given current economic and political trends, future electrical grids will require highly variable operations that impose significant technical and economic challenges for power producers. Introducing hybrid energy systems may help create a path to achieving highly variable markets that are economically sound and do not compromise grid reliability.

This paper presents background information on the methodology being developed to evaluate the economic merit of an NHES, with a focus on the development of dynamic multiphysics models in Modelica that play a key role in the economic evaluation. Additional information beyond the scope of this paper can be found in ORNL, 2016a, ORNL, 2016b, and ORNL, 2017. 


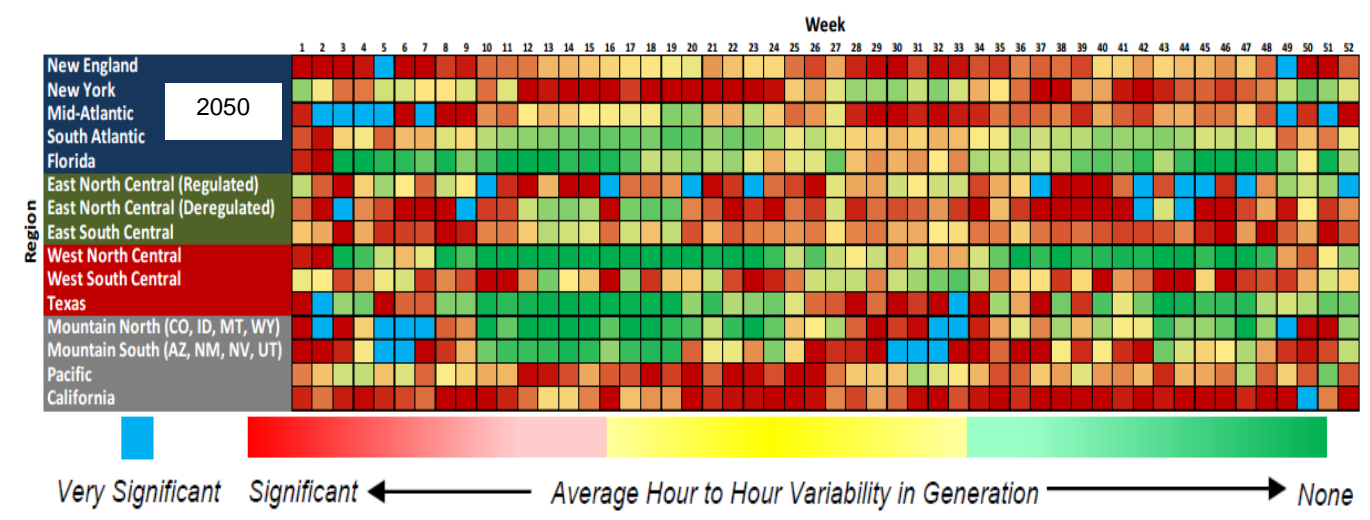

Figure 2. Prediction of electrical grid variability for regions of the United States in 2050. The color of the cells represents the variability. Regions approaching red and blue have demands that will be difficult and expensive for the electrical grid to meet-especially power producers operating under traditional market paradigms (EPRI, 2015).

\section{The Tightly Coupled NHES}

The reference hybrid energy system is referred to as a "tightly coupled" system. This coupling indicates that both the thermal and the electrical energy from the base load power supplier are integrated with one or more systems (e.g., industrial plant). The Modelica-based system under development is presented in Figure 3. The numbers in the figure correspond to the brief descriptions in Table 1.

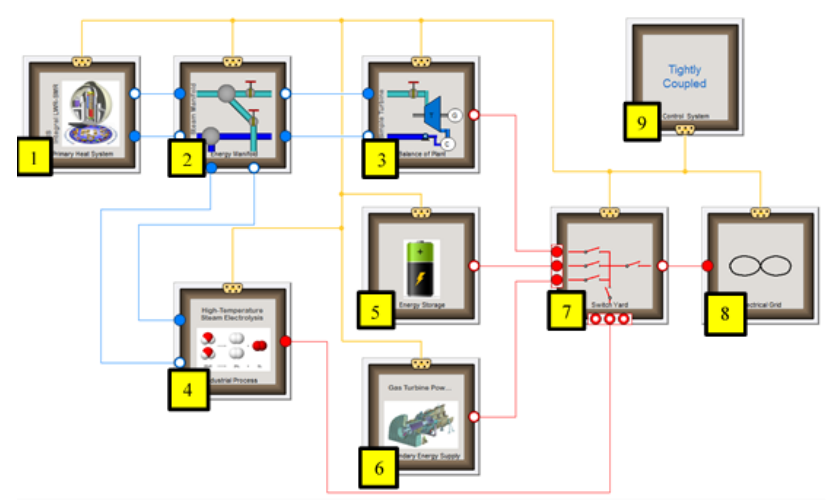

Figure 3. The tightly coupled NHES under development. The blue lines indicate fluid, the red lines indicate electricity, and the yellow lines indicate sensor/control signals.

The dynamic model is used to provide non-economic figures of merit - such as the ability to meet specified energy demands and overall system stability and reliability-to supplement the economic cost evaluation.
Table 1. Description of the various subsystems comprising a tightly coupled hybrid energy system.

\begin{tabular}{|c|l|l|l|}
\hline Identifier & Component & \multicolumn{1}{|c|}{ Description } & \multicolumn{1}{|c|}{ Example } \\
\hline $\mathbf{1}$ & $\begin{array}{l}\text { Primary Heat } \\
\text { System }\end{array}$ & $\begin{array}{l}\text { Baseload heat and } \\
\text { power }\end{array}$ & Nuclear reactor \\
\hline $\mathbf{2}$ & $\begin{array}{l}\text { Energy } \\
\text { Manifold }\end{array}$ & $\begin{array}{l}\text { Diverts energy to } \\
\text { subsystems }\end{array}$ & Steam distribution \\
\hline $\mathbf{3}$ & $\begin{array}{l}\text { Balance of } \\
\text { Plant }\end{array}$ & $\begin{array}{l}\text { Primary electricity } \\
\text { producer }\end{array}$ & Turbine and condenser \\
\hline $\mathbf{4}$ & $\begin{array}{l}\text { Industrial } \\
\text { Process }\end{array}$ & $\begin{array}{l}\text { Non-electric } \\
\text { commodity revenue } \\
\text { stream }\end{array}$ & $\begin{array}{l}\text { Steam electrolysis or } \\
\text { desalination }\end{array}$ \\
\hline $\mathbf{5}$ & $\begin{array}{l}\text { Energy } \\
\text { Storage }\end{array}$ & $\begin{array}{l}\text { Energy buffer to } \\
\text { increase overall } \\
\text { system robustness }\end{array}$ & Batteries and firebrick \\
\hline $\mathbf{6}$ & $\begin{array}{l}\text { Secondary } \\
\text { Energy }\end{array}$ & $\begin{array}{l}\text { Energy makeup } \\
\text { Electrical load }\end{array}$ & Gas turbine make-up \\
\hline $\mathbf{7}$ & Switchyard & $\begin{array}{l}\text { Electricity distribution } \\
\text { distributor }\end{array}$ & Electrical customer \\
\hline $\mathbf{8}$ & $\begin{array}{l}\text { Electrical } \\
\text { Grid }\end{array}$ & $\begin{array}{l}\text { Hub for sub-system } \\
\text { controls }\end{array}$ & $\begin{array}{l}\text { Control/supervisory } \\
\text { systems }\end{array}$ \\
\hline $\mathbf{9}$ & Control & & \\
\hline
\end{tabular}

\subsection{Economic Evaluation: Cost}

An economic evaluation of NHESs will be performed to investigate the minimum cost a hybrid system. This information informs decision makers on the planning and development of business/government agendas. To evaluate the economic cost of a given hybrid system, the Modelica model is coupled to the Reactor Analysis and Virtual control ENvironment (RAVEN), a multipurpose software framework developed by INL that allows for dispatching different software functionalities, including surrogate model generation and optimization routines (Rabiti et al., 2012). As outlined in Figure 4, RAVEN supplies the dynamic model demand time histories for specific subsystems along with subsystem capacities (e.g., industrial process production capacity). The system control logic then operates the overall system to meet the supplied demand. At the end of the simulation, various figures of merit (e.g., ability to meet demand, reliability based on operation of components) are passed to RAVEN. RAVEN then creates simplified surrogate models of the dynamic system and performs a cost-based optimization. This optimization generates 
new capacity parameters, and the process repeats until convergence to an optimized system is achieved. Using a high-performance computing cluster, this process is applied for many different cases in parallel.

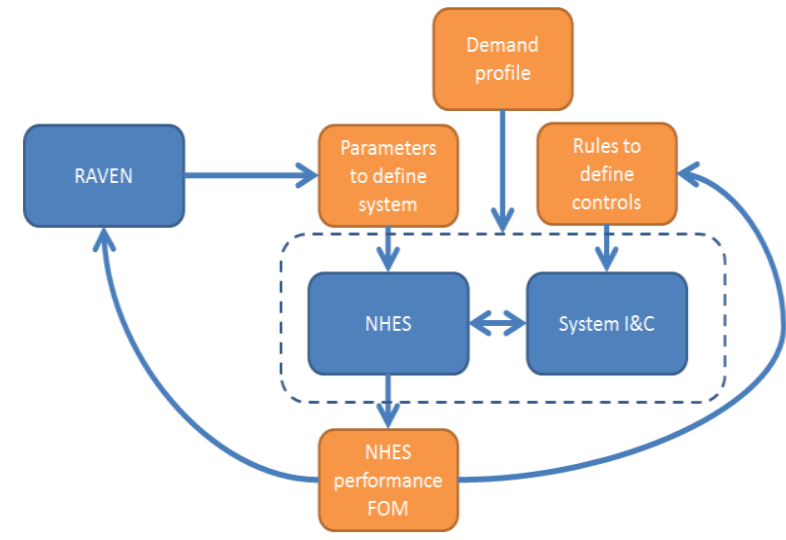

Figure 4. Modelica NHES dynamic model and RAVEN cost optimization process diagram (ORNL, 2016a).

\subsection{Electricity Demand Profile}

The specific energy demand profiles that provide set points to the dynamic model capture the variability of a specific energy market. For example, in a region with large solar power installations, the net electricity demand - consumer demand minus renewable supplyprofile would show significant reductions in demand in the middle of the day-the period with greatest insolation. Figure 5 demonstrates a characteristic demand profile and the associated contributions of each of an example set of power producers over the course of a year. The demand profile is fed to the Modelica model using the combitimetable component in the Modelica Standard Library (MSL), which uses a relative path to an external text file to enable operation on the cluster.

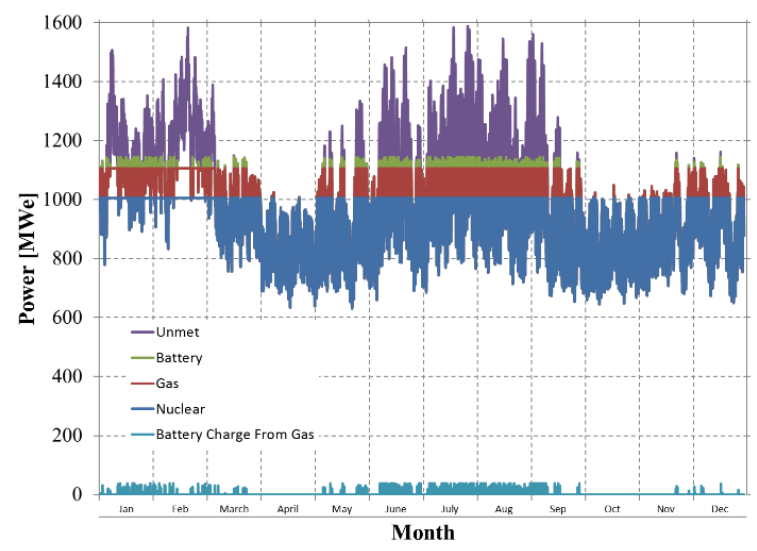

Figure 5. A one year electrical power demand profile characteristic taken from the north-east region of the United States (PJM, 2016). Each color represents the respective contribution of a subsystem energy supplier (ORNL, 2016b).

\section{Dynamic Subsystem Models}

Each of the subsystem models is built from a template, which allows for replaceable classes, improved interchangeability of control system approaches, and quick introductions of alternative subsystem models (e.g., replacing a steam electrolysis plant with a desalination plant). Figure 6 shows the template used when generating new subsystems and an example use case of the primary heat system. The subsystem models utilize the expandable connector signal bus for all control and sensor signals. Data records are also used to facilitate common reference values between subsystems, their control schemes, and the overall system.

$\square$ Template_SubSystem
Temp_example
$\square$ Dummy
$\square$ PartialSystem
$\square$ Partial_CS
$\square$ SignalSubBus
$\square$ DataNominal
四 DataInitial

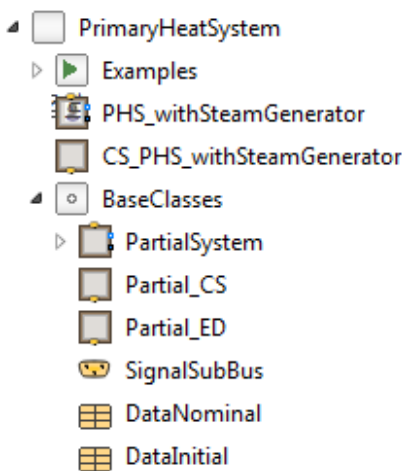

Figure 6. Subsystem template (left) and example use of the template (right).

\subsection{Example Subsystems}

In this section, the primary heat system, energy manifold, and balance of plant are briefly presented to better illustrate the physics-based modeling approach.

\subsubsection{Primary Heat System}

Figure 7 demonstrates the implementation of a primary heat source option, which - in this case - is an integral pressurized water nuclear reactor based on the International Reactor Innovative and Secure (IRIS) (Westinghouse, 2007). A few important physical phenomena captured in the model include the two phase dynamic interactions of the pressurizer, the generation of steam in a helical coil steam generator, and the behavior of a nuclear core. The nuclear core model is shown in Figure 8, and this model integrates the coolant flow geometry and behavior, fuel behavior, and point kinetics neutronics behavior, with feedback from the fuel and coolant temperature.

Models in the various subsystems use custom models, models from the MSL, and ThermoPower models. See Section 5 for more discussion on specific component modeling efforts. 


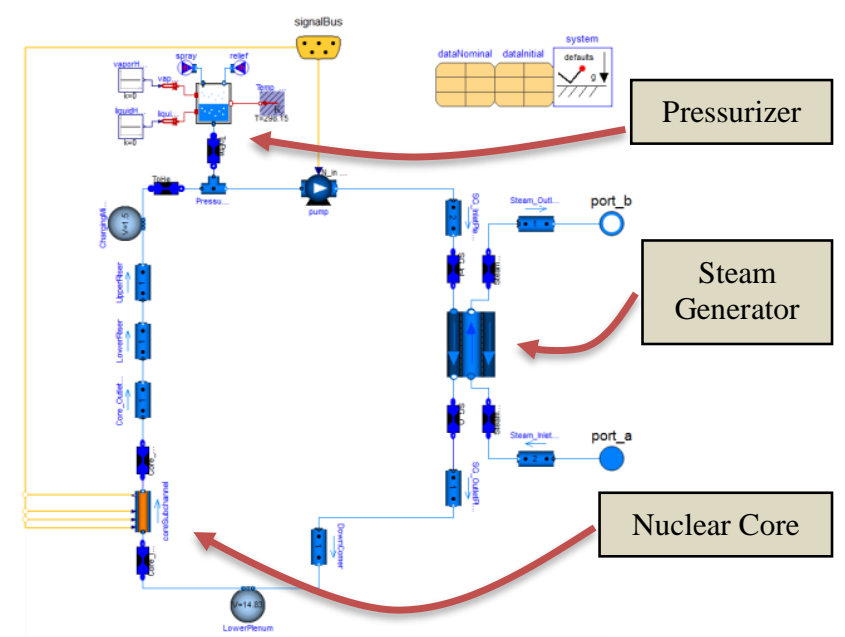

Figure 7. The Modelica model of the IRIS integral pressurized water nuclear reactor being used as the primary heat source subsystem.

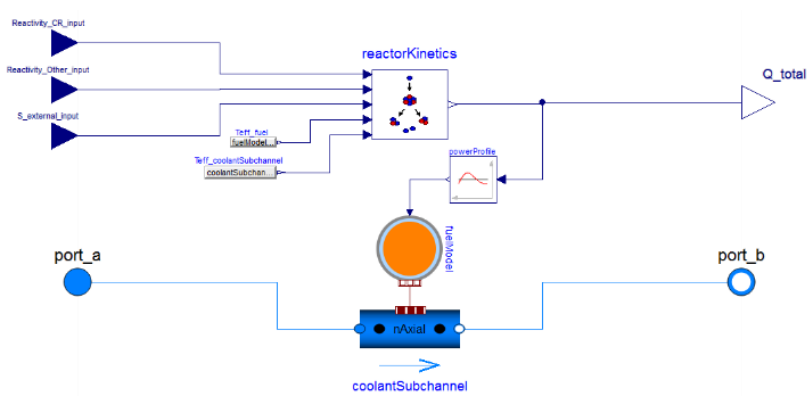

Figure 8. Model of a nuclear sub channel incorporating the neutronic behavior, non-uniform power generation, fuel conduction model, and coolant sub channel flow model.

\subsubsection{Energy Manifold}

The current distribution system under consideration is a purely thermal (i.e., steam/water) manifold (Figure 9). The energy manifold relies on controller logic to actuate distribution valves to handle large and slow power-set point changes to other subsystems, as specified by the demand profile. This actuation diverts hot steam coming from the primary heat system to the desired destination. The manifold also gathers return streams and directs the flow back the primary heat system steam generator at the proper temperature and pressure. Mixing and splitting volumes then add thermal mass to the system, dampening transient behaviors.

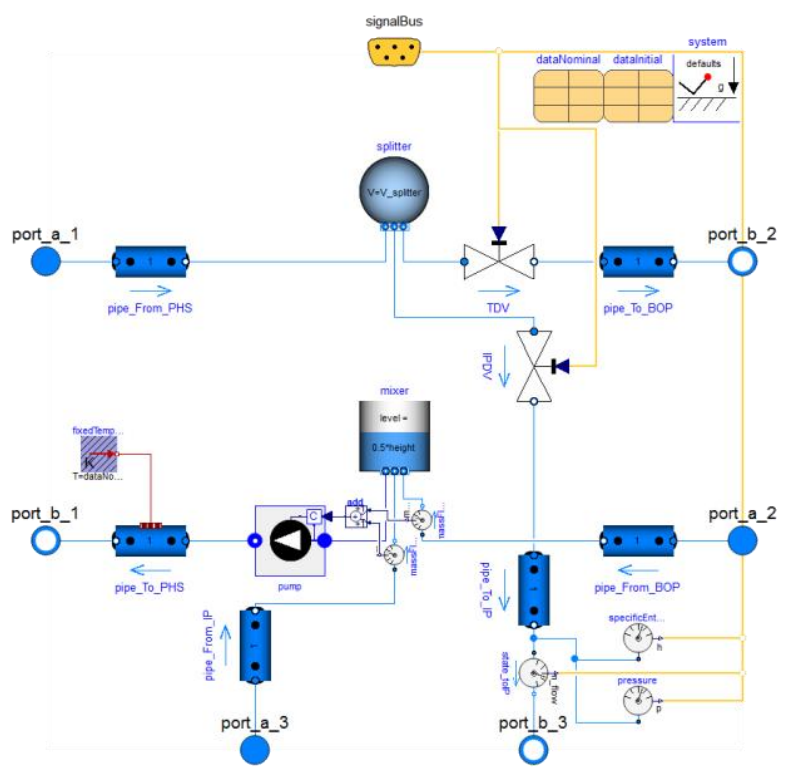

Figure 9. "Steam" energy manifold responsible for directing thermal energy to connected subsystem models.

\subsubsection{Balance of Plant}

One of the connections to the energy manifold is the balance of plant. The balance of plant is responsible for generating the primary share of electrical energy in the hybrid system. The current, simple model contains a steam turbine for electrical power generation, a condenser, and a control valve (Figure 10). The turbine control valve is responsible for small, fast control modulations.

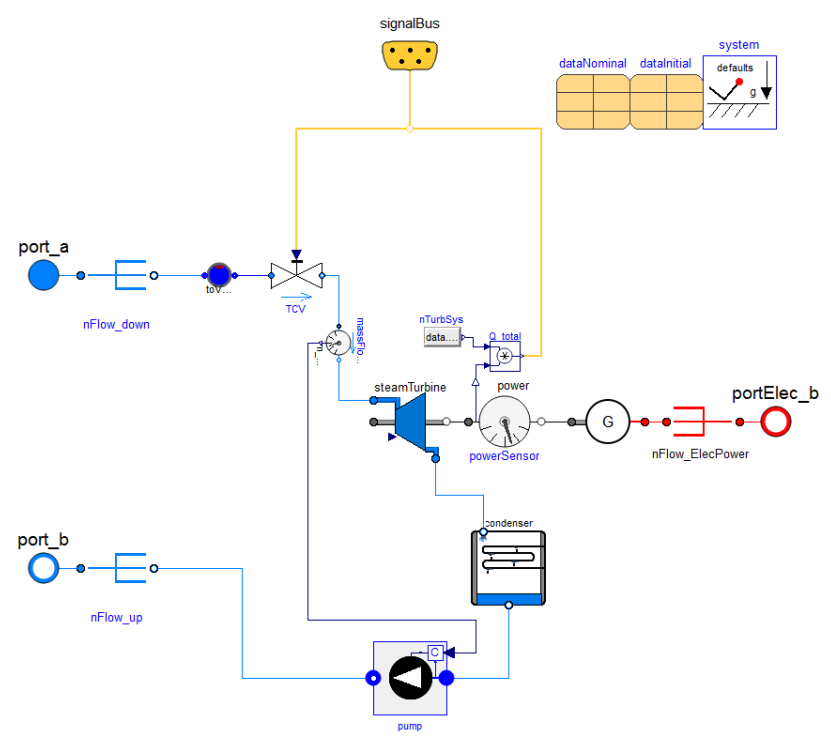

Figure 10. Simple balance of plant model, which converts steam thermal energy to electrical energy and returns subcooled water back to the energy manifold. 


\section{Preliminary Model Performance}

A preliminary testing of the model reads the external data that contains the time series electrical demand profile and then feeds this data to the balance of plant control system. The control system actuates the control valve to match demand as much as the physical process allows. Figure 11 demonstrates 10 hours of dynamic turbine power operation based on a demand profile. The power changes are accomplished via the manipulation of actuators such as the turbine control valve position. At approximately hour three, the power set point is above the deliverable power. Situations like this period of unmet demand are tracked to inform the economic evaluation.

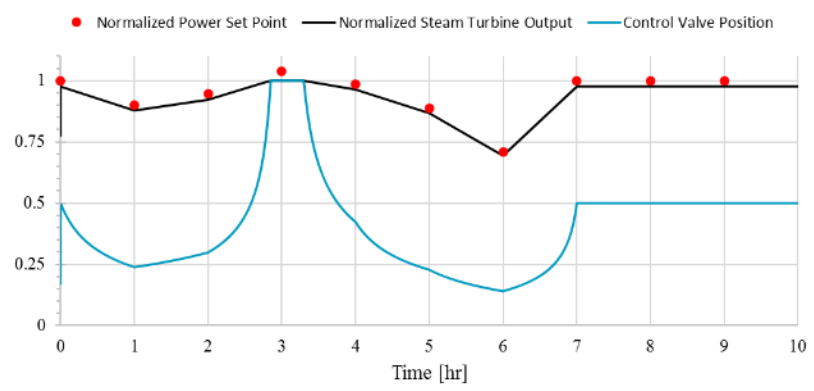

Figure 11. Preliminary test case demonstrating the ability of the coupled hybrid system to track a variable electrical demand profile by diverting flow to/from the steam turbine. Note the unmet demand at hour three.

The current NHES model consists of 14,581 equations and simulates a one-week period in approximately 2 hours using Dymola 2017 FD01 on a desktop computer (16 GB ram, Intel Xeon CPU ES-1607 v3 3.10GHz). Figure 12 presents the set point and measured electrical production values of a week-long simulation.
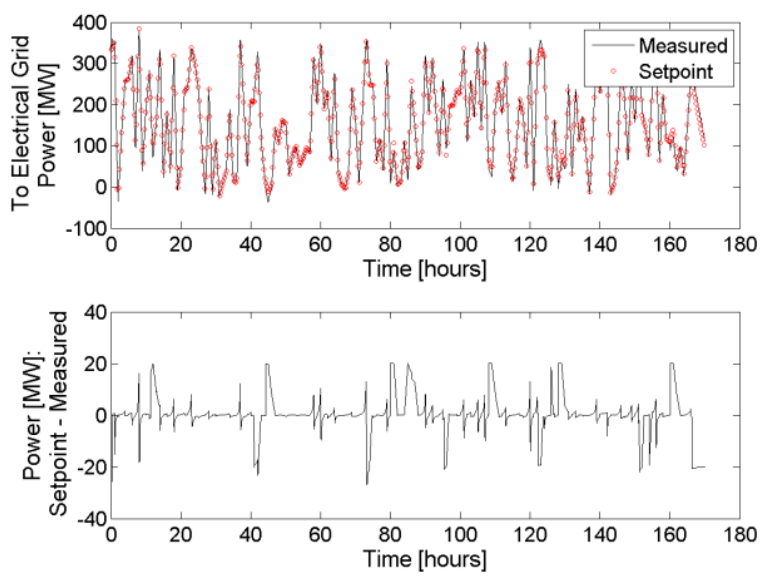

Figure 12. Load following electrical power production from the NHES model over a period of one week.

\section{Component Development}

The modeling activity uses components and connectors from the MSL along with a few components from the
ThermoPower library. However, user experience has identified various limitations to some components. Therefore, several components have been improved or remade for the needs of this project. A brief discussion of two major components are presented in this section.

\subsection{MSL: Dynamic Pipe to GenericPipe}

There are many positive aspects of the current version of the MSL DynamicPipe model. For example, the flexibility of specifying the model structure and the ability to easily change the number of discretized volumes, flow, and heat transfer models is incredibly useful. However, some significant limitations were discovered when attempting to couple the dynamic pipe with fuel and reactor neutronics models.

One primary issue was the inability to specify temperatures or enthalpy distribution for the start values of each control volume. The current DynamicPipe assumes a linear distribution between the ports. Since the neutronics model is highly sensitive to the temperature of the coolant and fuel, simulations often failed during the initial transient phase due to extreme power fluctuations in the reactor core.

To more generalize the capabilities of a pipe model, a new GenericPipe model was created. This model is similar in structure to the DynamicPipe model, but it removes some of its restrictions (e.g., added control of initialization and geometry) and works towards a more standard, organized approach to model development. Figure 13-Figure 15 show a few parameter windows displaying the new controls of GenericPipe along with the modified structure for various closure models, including heat transfer, pressure loss, and geometry.

This generic pipe can also be used to create simpler versions with more refined parameters-including DynamicPipe-to ease user interaction. For comparison, a few examples provided in the MSL fluid package (e.g., BranchingDynamicPipes) were recreated using the GenericPipe model and then benchmarked. Current tests using GenericPipe yield the same solutions as the DynamicPipe model but with computational speeds up to $30 \%$ faster.

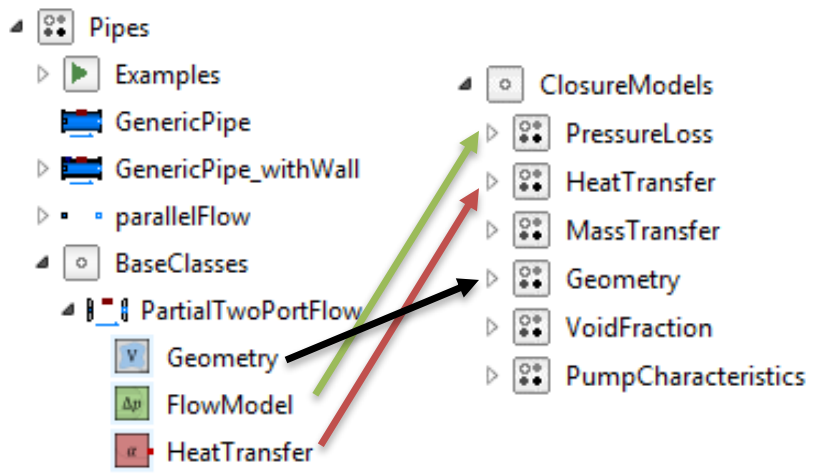

Figure 13. Structure of the generic pipe model demonstrating the expanded flexibility of the model. 


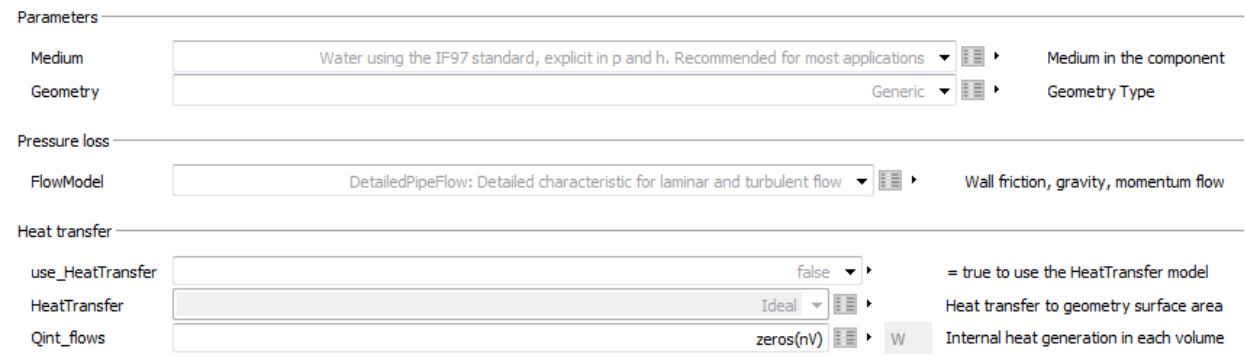

Figure 14. "General" parameter tab of the generic pipe. Note the "Geometry" parameter allows for replaceable geometries (e.g., straight pipe and shell and tube or plate heat exchanger).

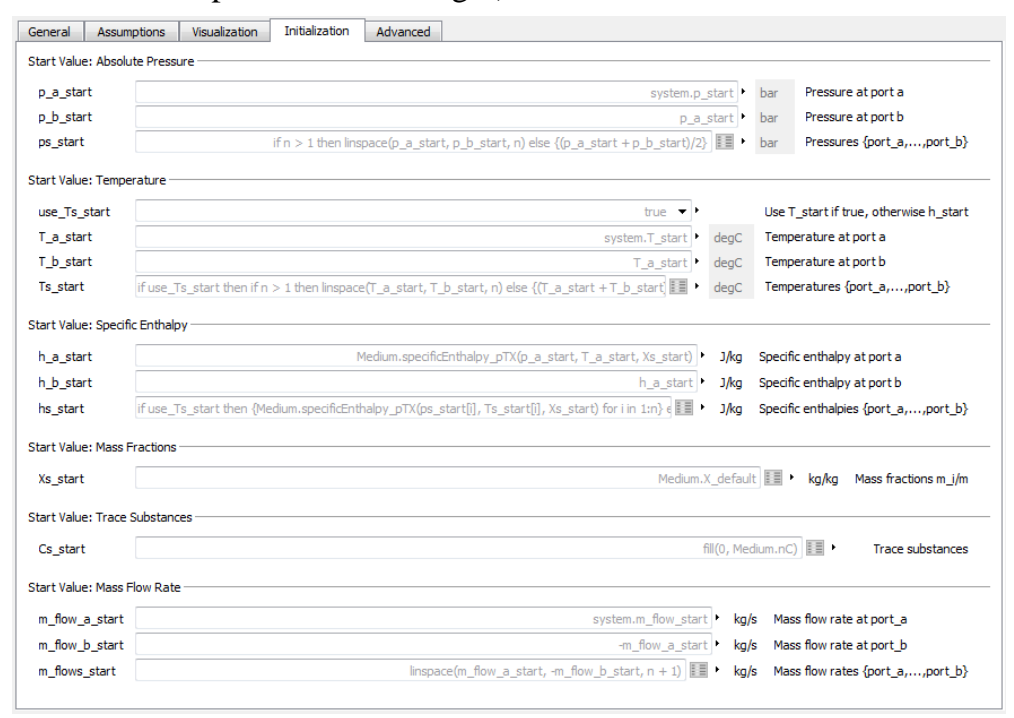

Figure 15. Improved initialization control for the pipe model permits simple initialization schemes based on port values or more precise schemes based on discretized volume states.

\subsection{Custom: Thermal Library}

The temperature response of a system is very important, particularly in nuclear reactors. The nuclear fuel temperature impacts not only the coolant flow behavior but also the power of the reactor itself by altering the behavior of the neutronics. To produce reasonably accurate models of nuclear fuel, a generic multidimensional discretized conduction model was created.

As part of this effort, the MSL Thermal package was completely redone to create a standalone library, which also includes a package of thermal resistances for steady-state evaluations, fin efficiency calculations, etc. (Figure 16). The created models are generic and can be

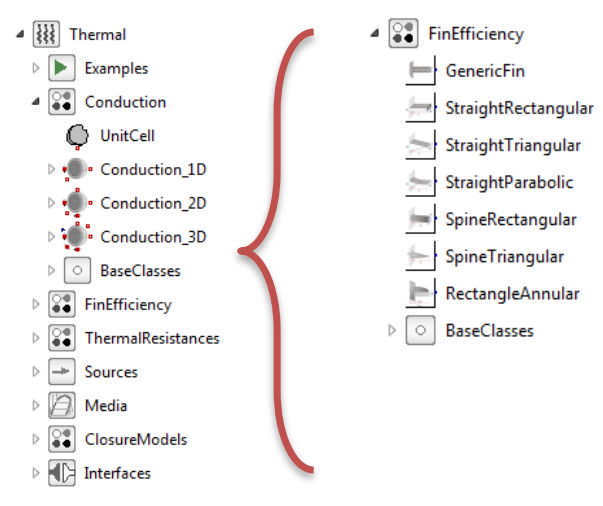

incorporated into cases that require thermal inertia or dynamics of conduction in solids. An important aspect of the library is the limited application of parameters to only those variables which require the variable type (e.g., initialization variables). All other parameters are specified as type input to ensure the user has maximum flexibility in model development. Additional features such as radiation models will be added to the Thermal package in the future.

\subsubsection{Multi-Dimensional Conduction Models}

Given the complex nature of multi-dimensional models, additional discussion on a conduction model is presented. Three different approaches were evaluated in

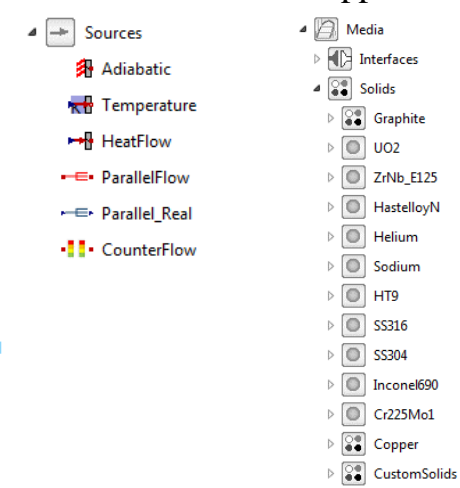

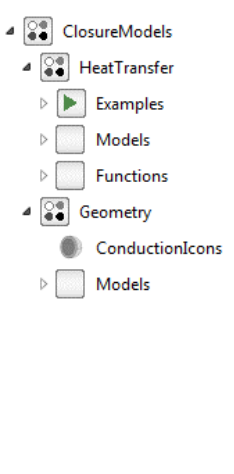

Figure 16. Thermal library with multi-dimensional conduction models, thermal resistance models, etc. 
developing the conduction models: the "classical", "modelica", and "mixed" approach.

The classical programming approach relied on a replaceable "solution method" that defined the connections between cells. This approach has limited flexibility as equations (e.g., spatial differentiation of the energy equation) are hard-coded to initial assumptions such as geometry.

The modelica approach relied on independent, singlenode, models to specify the behavior of unit volumes and the energy flow between cells; these models were then connected using connect () statements. Figure 17 shows the diagram layer of this modelica method and depicts the use of simpler models to build up more complex models.

The mixed approach limits use of connect () and instead applies models that are have built-in nodalization which allows direct assignment of the variables that must be shared between models. In other words, the mixed method attempts to hard-code all generally applicable features of the model and only rely on the Modelica generated equations/connections when necessary while avoiding the embedded assumptions of the classical approach.

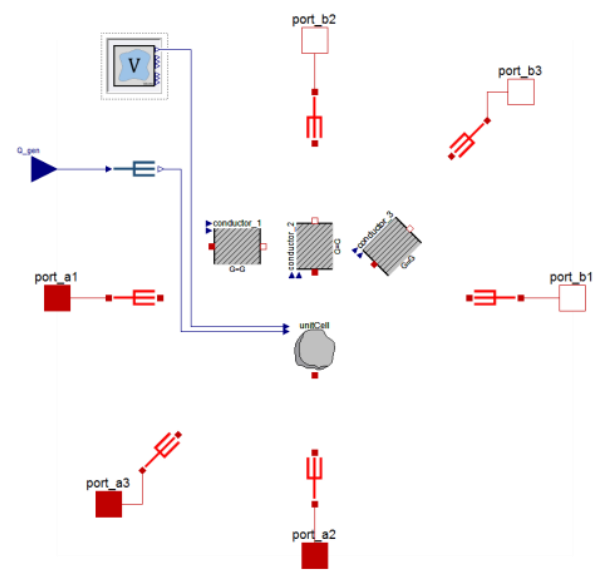

Figure 17. Diagram layer of Conduction_123D using the modelica approach. This method creates multidimensional conduction models by using independent models to build more complex, standardized models.

Each of the approaches have successfully modeled the needs of the hybrid energy system (e.g., fuel element modeling and heat exchanger walls). Figure 18 shows a surface plot of a fuel model with a fuel, gas gap, and cladding region created using the conduction models. Each of the regions have temperature-dependent properties specified by the solid media package.

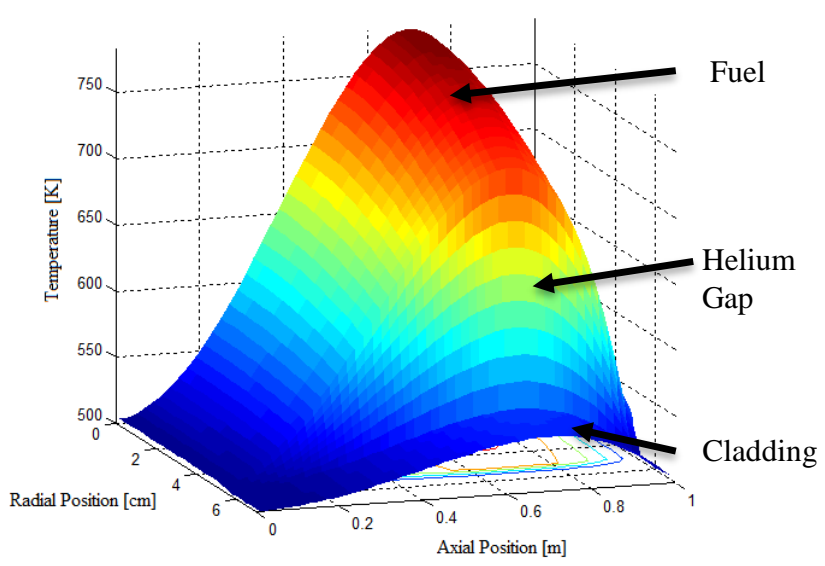

Figure 18. Surface plot of a non-uniformly heated fuel element (fuel, gap, and clad) with external convection created using the discretized conduction models.

Comparisons have shown that all three approaches produce results comparable within a small and reasonable margin of error (fractions of a degree Kelvin), however, the computational resources of the three approaches vary significantly. The classical approach passes the translation process quickly-even for a large number of discretizations - and then simulates quickly. The modelica approach can complete a simulation in similar or less time than the classical approach; however, the time it takes for the modelica approach to translate the model becomes more significant as the number of nodes being used increases (Figure 19).

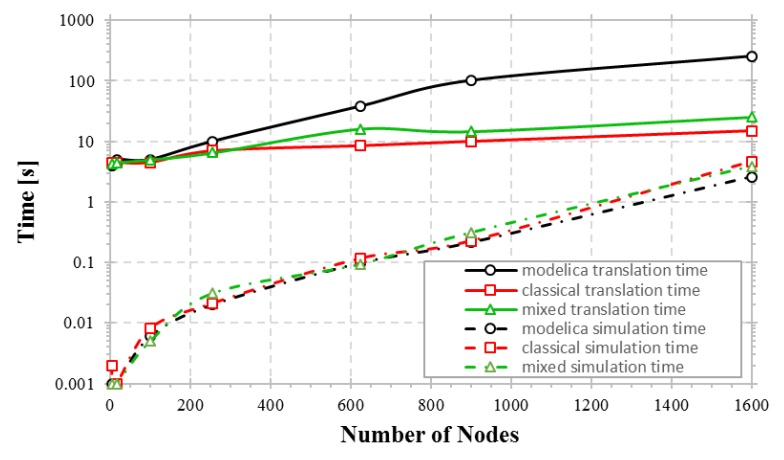

Figure 19. Demonstration of the translation and simulation times required for each of the methods of a discretized conduction model. For a given number of nodes, the modelica method requires far more translation time than the classical or mixed method, whereas the simulation times for each method remain similar.

The issue of translation time stems is primarily a result of relying on connect (), and therefore the translator, to generate the necessary equations for the solver. Figure 19 demonstrates that for a fixed number of many equations (e.g., 90,000), the classical approach-as compared to the modelica method - can achieve a much finer discretization scheme (6,100 vs. 1,500 nodes) without compromising the translation time ( $50 \mathrm{~s}$ vs. 240 s). However, the mixed approach also generates $\sim 90,000$ 
equations with 1,500 nodes but passes through the translation phase in $25 \mathrm{~s}$. This demonstrates that the method in which the equations are generated, rather than just the number of equations, is the primary controller of translational time. The mixed approach resolves the translational time penalty while also preserving efficient simulations.

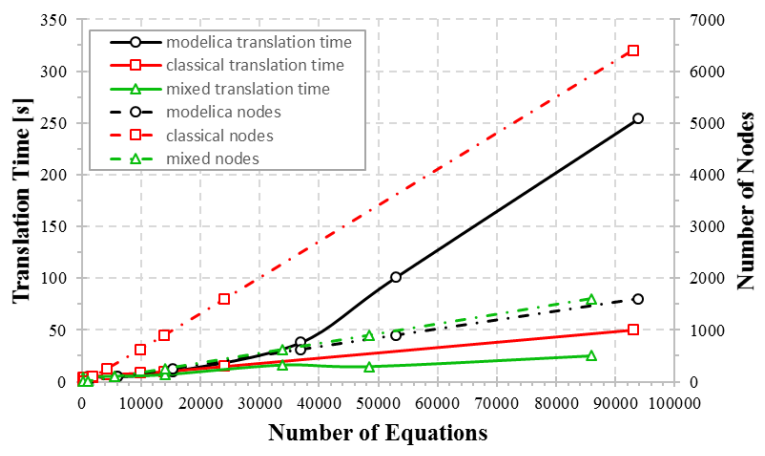

Figure 20. Demonstration of the relationship between the number of equations generated, the discretization scheme, and the required translation time for each of the discretized conduction model methods. The method of equation generation is the primary controller of translational time.

Although the modelica method adopts the best practices of Modelica programming by not repeating code, the ability to address the time for translation required the use of an alternative mixed approach. The findings of this study are important for the development of any discretized model and will be applied to additional physics of interest such as fluid flows and neutron behavior.

\section{Conclusion}

As energy markets shift to a highly variable demand profile, traditional base load power suppliers will be required to modify their business models. A hybrid energy system approach, coupling base load energy suppliers and energy customers (thermal and/or electric), may be profitable and preferred in future energy markets. The detailed dynamic multi-physics models discussed in this paper are being coupled to an economic cost optimization study that will inform the potential benefits and limitations of these hybrid systems by providing critical dynamic physical data of a potential hybrid system's operation.

As part of NHESs development, various components models are required to capture the important physical responses of the system. Two key models are the pipe model and thermal conduction models. This paper discussed adaptations and improvements to a Generic Pipe model and the creation of a new Thermal library. The thermal library includes multi-dimensional conduction models. Using these conduction models, an investigation of proper model formulation has been performed demonstrating a methodology to maximize model flexibility while retaining computational efficiency.

\section{Acknowledgments}

This project was funded by the US Department of Energy's Office of Nuclear Energy under the Office of Advanced Reactor Deployment.

\section{References}

Bragg-Sitton, S.M., R. Boardman, M. Ruth, O. Zinaman, C. Forsberg. 2015. Rethinking the Future Grid: Integrated Nuclear Renewable Energy Systems. Report no. NREL/CP6A20-63207.

EPRI (Electric Power Research Institute). 2015. Program on Technology Innovation: Fossil Fleet Transition with Fuel Changes and Large Scale Variable Renewable Integration. Technical report no. 3002006517.

ORNL (Oak Ridge National Laboratory). 2016a. Nuclear Hybrid Energy System FY16 Modeling Efforts at ORNL. Report no. ORNL/TM-2016/418. Oak Ridge, TN.

ORNL (Oak Ridge National Laboratory). 2016b. Nuclear Hybrid Energy System Initial Integrated Case Study Development and Analysis. Report no. ORNL/TM2016/707. Oak Ridge, TN.

ORNL (Oak Ridge National Laboratory). 2017. Nuclear Hybrid Energy System Model Stability Testing. Report no. ORNL/TM-2017/153. Oak Ridge, TN.

PJM. 2016. Estimated Hourly Load. Accessed November 4. http://www.pjm.com/markets-and-operations/energy/realtime/loadhryr.aspx.

Rabiti, C., A. Alfonsi, J. Cogliati, D. Mandelli, and R. Kinoshita. 2012. Reactor Analysis and Virtual control ENvironment (RAVEN), FY12 report. Technical report no. INL/EXT-12-27351. Idaho Falls, ID: Idaho National Laboratory (INL).

Westinghouse. 2007. Computer Models for IRIS Control System Transient Analysis. Report no. STD-AR-06-04. 